\title{
Assist Driving Control System based on Vehicle Networking
}

\author{
Weimin Zhu \\ Postgraduate \\ Shanghai University of \\ Engineering Science, \\ Shanghai 201620, China
}

\author{
Jin Liu* \\ Adjunct Professor \\ the correspondence author \\ Shanghai University of \\ Engineering Science, \\ Shanghai 201620, China
}

\author{
Meng Li \\ Postgraduate \\ Shanghai University of \\ Engineering Science, \\ Shanghai 201620, China
}

\begin{abstract}
As the major intersection of Internet of Things (IoT) and smart car, Internet of Vehicles, is a specific application in Internet of Things. Especially nowadays more and more people concerned about the issue of traffic safety, so how to reduce or avoid traffic accidents by Internet of Vehicles technology, has become the most important and the most urgent problems to be solved in the development of Internet of Vehicles. RFID and ZigBee technologies are applied to get the distance and speed information between the smart cars, with scientific strategies to control driving distance, the system can keep the cars between safe distances and be able to make timely deceleration, braking and other treatment before the accident and other emergency situations. This automatic driving and warning system is helpful to solve some of the existing problems. At the same time, through the analysis of problems in the debugging process, optimization scheme is proposed to improve the reliability and safety of the assist in driving system.
\end{abstract}

\section{General Terms}

ITS ; Internet of Vehicle; PC; Monitoring system.

\section{Keywords}

ITS, Internet of Vehicle, RFID, ZigBee, Assist in Driving

\section{INTRODUCTION}

Traffic safety has been the focus of attention and thinking, it is widely concerned by governments around the world. Every year in the world, traffic accidents cause huge losses. Also with the increase of the number of private cars and the development of the highway, car flow will gradually increase, and the speed will continue to improve. Along with the growth of these data, it will expose a series of man-made factors and thus leads to traffic accidents occur frequently. Although the parties have taken many safety precautions to avoid or reduce the probability of occurrence of traffic accidents, the basic reason that causes the traffic accident to occur is not resolved. In the future, the development of the vehicle networking technology will help to solve the traffic safety problem. The development of research in real-time access to the road or vehicle information to help drive control the car, therefore, become the fundamental way to solve the problem of traffic safety.

By drawing on the concept of the Internet technology in the current rapid development, a driving control system based on vehicle networking is designed. The system through the ZigBee, RFID and other technology to obtain the vehicle in the driving process of the vehicle's speed and relative distance, and it is based on the reasonable vehicle distance strategy, making timely anticipation and response, hoping to avoid the occurrence of traffic accidents.

\section{SYSTEM HARDWARE DESIGN 2.1 Intelligent Traffic Sand Table Model and Application}

A reliable information transmission path is established between the vehicle and the vehicle, and the whole city traffic is included in a huge network. Like the rise of the Internet in the last century 80 's, the world has experienced a great revolution, car networking will also be doomed to change the future of people's work, life style.

In order to explore and develop the intelligent transportation and vehicle networking technology, the system introduced a networking intelligent transportation system in the form of sand table simulation intelligent transportation application scenarios show. The system used the technology of embedded, RFID, sensor detection, WiFi transmission, Java, Android, etc., and integrates the current Internet of things technology and transportation industry. The design of the vehicle based on the help of the driving control system, this article mainly introduces the hardware from the two aspects of the smart car and sand Lane. In order to realize the intelligent traffic control system, the speed and distance are two parameters that need attention. The system obtains the intelligent traffic sand table model of intelligent vehicle and the distance between the smart car speed by using RFID and ZigBee technology, with the science of vehicle distance strategy, to achieve adaptive adjustment between the vehicle speed, to maintain a safe distance, and when unexpected situations occur and other unexpected situations, the system can timely make a reduction, braking, etc.. The automatic driving warning system based on the Internet of things technology can make up some deficiencies in the current adaptive cruise system, and it is hoped that the combination of the two systems can make up for the deficiency.

\subsection{Intelligent Car System Scheme}

The model of the smart car comes from the Freescale smart car competition, on its basis, RFID and ZigBee technology of the Internet of things is added. The hardware control system frame is shown in figure 1. 


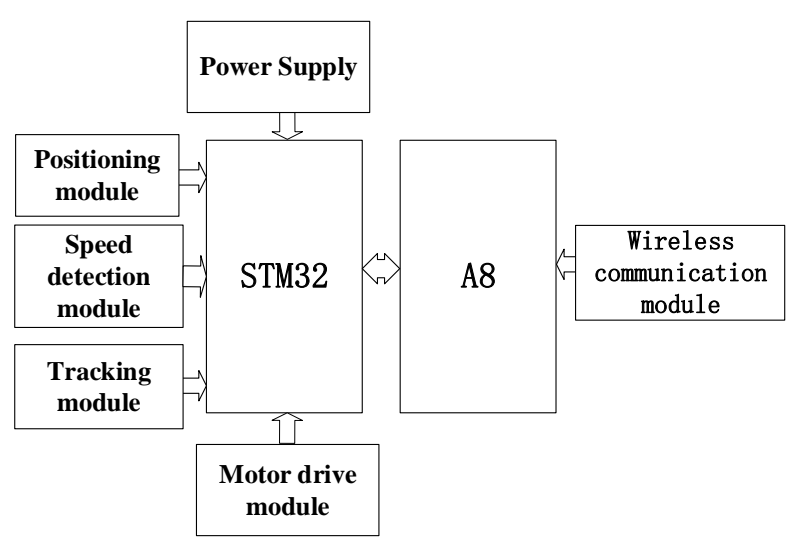

Fig 1: Hardware Control System Block Diagram

The function of each module is as follows:

(1) Control system module: responsible for the information receiving, data processing, and according to the corresponding command to control the various modules of the smart car to complete different tasks.

(2) Power module: responsible for the entire intelligent car to provide a variety of power supply.

(3) Positioning module: responsible for the acquisition of smart car in the sandbox position.

(4) Speed detection module: responsible for the acquisition of smart car driving speed.

(5) The tracking module: it is responsible for the current signal of intelligent car following driving lane laid along the sand.

(6) Motor drive module: responsible for the output of the microcontroller PWM wave signal, so that the DC motor work in the setting of the state.

(7) Wireless communication module: the wireless communication module for communication between the sandbox smart car, smart car to get the speed and distance information.

\subsection{Control System}

According to the application domain of the microprocessor, microprocessors can be roughly divided into three categories: General purpose high performance microprocessor, embedded microprocessor and digital signal processor, microcontroller. Smart car designed, in addition to the control system to achieve the basic movement of the car, also need to process data in real time, multi thread, multi task coordination and cooperation to complete the car networking strategy control. Therefore, the smart car needs to be controlled by the two layer control board. The bottom board is used to select the high performance micro controller (MCU) as the vehicle's motion control unit with rich IO interface resources and peripheral resources. Advanced version chooses multithreading, excellent operating rate of embedded microprocessors (ARM) as a vehicle networking and real-time strategy to respond to the control unit.

\subsection{Power Module}

Smart car requires three different working voltages: $7.2 \mathrm{~V}, 5 \mathrm{~V}$ and $3.3 \mathrm{~V}$. Among them, the motor drive module needs to use $7.2 \mathrm{~V}$ and $5 \mathrm{~V}$ two kinds of operating voltage. Positioning module, speed detection module, wireless communication module and the steering gear needs to use the $5 \mathrm{~V}$ operating voltage. The micro controller module, tracking module and serial port needs to use $3.3 \mathrm{~V}$ operating voltage.

\subsection{Motor Drive Module}

Motor driver module use BTS7970B half bridge motor driver chip. The motor drive chip can be directly used to drive some of the high power inductive loads, such as DC motor, stepper motor and relay, etc. The polarity conversion of the motor driving voltage can be realized by the logic setting of the input terminal of the chip control signal input. That is to realize the adjustment of direction change.

\subsection{Speed Detection Module}

The driving speed of the intelligent vehicle is controlled by the closed-loop PID, and the speed feedback is obtained by using the OMRON speed coder. The digital signal of A and B is captured by the timer input. When the A phase signal is high power, comparing the B phase is high or low level, so as to determine the motor is being or reverse, that is to get the direction of the intelligent car. At the same time, the number of the output pulses of the unit time $(10 \mathrm{~ms})$ encoder output pulse is counted by using the timer, and then the speed of the motor is calculated.

\subsection{Tracking Module}

By using the electromagnetic tracking method, the smart car can realize automatic path detection. The sand below each lane is paved with a $20 \mathrm{KHz}$ alternating current signal. By installing the sensor in front of the smart car, the magnitude of the induced electromotive force can be detected. And the collected data are processed by the corresponding algorithm, so as to realize the automatic path detection of intelligent car.

\subsection{Positioning Module}

Table top positioning uses RFID Technology, intelligent sand Lane every $15 \mathrm{~cm}$ is laid at the bottom of an electronic tag. Each tag is recorded in the table position. By integrating the RFID circuit in the bottom of the car STM32 control board, you can read the stored in the electronic tags inside the location information, so you can get the current position of the car.

\subsection{Wireless Communication Module}

Intelligent car using ZigBee technology to achieve the wireless communication between the car. ZigBee core control part uses the production of TI CC2530 chip, which is embedded in the microcontroller and wireless transceiver module. In addition, in order to improve the output power and gain of the wireless communication module, the RF front-end amplifier with RFE2401C is adopted, RF front-end amplifier which features compared with other similar chip, the external circuit is very simple, which greatly simplifies the design of RF circuits.

\section{SOFTWARE SYSTEM DESIGN}

Smart car dashboard underlying task is to complete initialization and drive the car of each hardware module, including motor drive module, speed detection module, a tracking module and positioning module. Smart car advanced control panel's mission is to achieve ZigBee wireless communication capabilities, processing and response trolley spacing strategy. By sending a control panel up from the bottom of the vehicle speed and position information, advanced control panel will compare this information with the previous vehicle speed and position information, and compares the results into the pitch control algorithm, the pitch 
control algorithm outputs different instructions depending on the circumstances, advanced control panel will send instructions back to the bottom panel, the bottom of the control panel in accordance with instructions received, adjusted driving state car. The overall program structure of the smart car a block diagram is shown in Figure 2.

\begin{tabular}{|c|c|c|}
\hline $\begin{array}{c}\text { Wireless } \\
\text { communication } \\
\text { module }\end{array}$ & $\begin{array}{l}\text { Strategy } \\
\text { control }\end{array}$ & $\begin{array}{c}\text { Advanced } \\
\text { control } \\
\text { panel }\end{array}$ \\
\hline $\begin{array}{c}\text { Speed detection } \\
\text { module }\end{array}$ & $\begin{array}{c}\text { Motor drive } \\
\text { module }\end{array}$ & \multirow{2}{*}{$\begin{array}{c}\text { Bottom } \\
\text { control } \\
\text { panel }\end{array}$} \\
\hline $\begin{array}{l}\text { Tracking } \\
\text { module }\end{array}$ & $\begin{array}{c}\text { Positioning } \\
\text { module }\end{array}$ & \\
\hline
\end{tabular}

Fig 2: Overall program structure diagram of smart car

\subsection{Wireless Communication Module Program Design}

Smart car uses ZigBee mesh networking between broadcast communications, every time smart car read a new RFID tag, ZigBee module will be broadcast once, and broadcasting is according to CSMA / CD Sense Multiple Access / Collision Detection Technology. Specific workflow is: Uploading underlying Dashboard update RFID tag information; advanced control panel for data encapsulation process, sending encapsulated data to the ZigBee module; ZigBee module query the current usage of the channel, if it is idle, a connection is established, then transmit data. If the channel is occupied, the system would delay period of time, then testing whether the channel is idle. The wireless communication module program flow chart is shown in Figure 3.

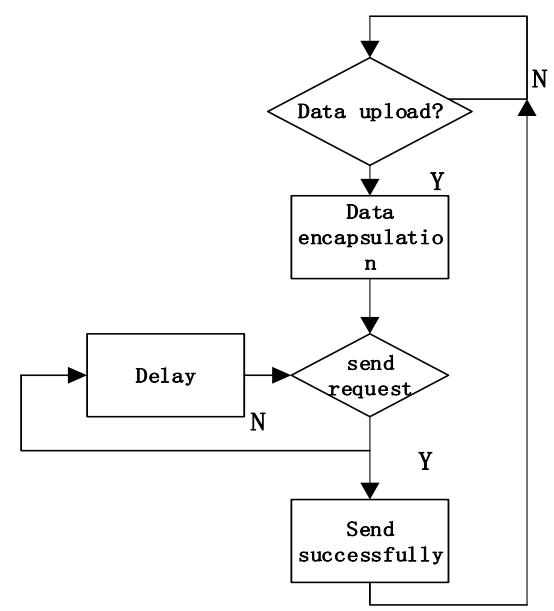

Fig3: Wireless communication module program flow chart

\subsection{Design of Spacing Policy Control Program}

Smart car warning system autopilot pitch strategy is to compare front vehicle speed information and distance information and the vehicle information, depending on the circumstances, the pitch is divided between the front of the car and the vehicle into four areas: brakes region, deceleration region, cruising area and the acceleration region.

When the smart car is in the acceleration region, indicating that the distance between the car at this time of great intelligence, security risk of rear-end collision does not exist. In this case the speed is too slow, it should increase the speed of the cars according to the vehicle in front.

When the smart car is within the cruising area, indicating that this time from the smart car is kept in the most appropriate range, the risk of a rear-end collision safety does not exist. It should make the smart car in front of the car at the same speed and follow state, according to the vehicle in front of the vehicle speed setting smart car speed.

When the smart car is within the deceleration region, indicating the distance between this case the smart car is reduced, the security risk of rear-end collision occurred existence. Smart car should be allowed to slow down, reducing the speed of the cars of the smart car speed according to the vehicle in front.

When the smart car is in the braking area, indicating that at this time the distance between the smart car has less than safe driving distance, smart car and the vehicle in front there is a security risk of rear-end collisions, in order to avoid rear-end collision, then the smart car should immediately set the speed zero.

\section{DEBUGGING AND OPTIMIZATION OF SYSTEM}

In order to verify the feasibility of assist driving control system in space strategy, using two sets of smart car to debug in the sand. Through the simulation of four kinds of space strategy, testing whether or not the smart car has made a correct response. Test method: on the one hand, through the observation of two intelligent car running in the sandbox state of subjective judgment of the smart car is under different situation made corresponding adjustment, on the other hand, running state information of the smart car remote printing on a $\mathrm{PC}$, including the car and the car in front of the distance (node), before the car speed (front) and the vehicle speed (self), making an objective judgment of validity about the program execution. The next is analysis of the data of the smart car remote printing.

\subsection{Results of System Debugging}

In order to accelerate the regional response as an example, according to the setting of the spacing strategy, while the intelligent car is in the acceleration area, the speed of intelligent car should be increased according to the speed of the car in front. According to the procedure, when the distance between two cars is more than 25 less than or equal to 35 , the latter is in the acceleration area, the speed of the latter vehicle should be plus 50 based on the speed of the car in front. As the data of debugging in acceleration area shown in Figure 4, the car that is running behind is stopping motion at first, once the distance between the cars falls in the acceleration area, the latter begins to run. At this time, the speed of the car in front is 90 , the speed of the latter is 140 , and the correctness of the accelerated regional subprogram has been verified. 


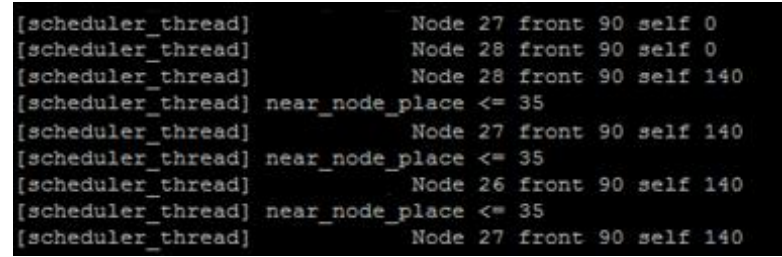

Fig 4: Acceleration zone debug data

\subsection{Problems of System Debugging}

After the verification of the validity of two intelligent cars in a low speed mode, improving the running speed of the intelligent car, and increasing the number of smart car on the sand table to test whether the system can also operate normally. In the process of debugging, it is found that after the intelligent car running for a period of time, the received data will fluctuates, resulting in miscarriage of justice, such as while the car in front is driving normally, the latter may take a sudden stop.

After the exclusion of the problem of spacing strategy, it is found that the delay caused by ZigBee communication, which leads to the lost of real-time speed of the intelligent car and distance information. Obviously, as a system to assist the driving control in intelligent transportation, the problem is absolutely not allowed. Therefore, it is necessary to analyze the causes of the problem, to find the corresponding solution or program, so as to enhance the security and reliability of the system.

\subsection{Solution}

The solutions are mainly from two aspects: the optimizing of the disposition of information and the transmission process of information of the optimized ZigBee communication system.

For the problem that delay of information disposition caused by the intelligent car faster, the broadcast cycle shorter, this information can include through information received by each radio frame transmission time screened data frames of the latest time processing, and the expired data frame directly discarded; aiming at the problems of sand on the smart car number increased after the delay information is intensified, by identifying each ZigBee radio data frame contains the vehicle ID information, several intelligent car ID number will distance car closer to the corresponding data frame processing to raise the priority, from and alleviate the increase in the number of cars caused by random delay.
Using the technology of time division multiple access and frequency points multiple access to allocate a time slot to each intelligent car and make the ZigBee module intelligent car work on several different frequency points, which can greatly reduce the influence caused by random error.

\section{CONCLUSION}

Through actual debugging in intelligent traffic sand table, respectively, smart car were conducted in the accelerated region, cruise area, deceleration area and brake area of subjective and objective tests. The test results verify the correctness of the vehicle based on vehicle network to assist the driving control system; At the same time, in the process of debugging, it is found that the information delay caused by ZigBee network topology structure and information processing, So as to put forward the corresponding information processing optimization and information transfer process optimization program.

\section{REFERENCES}

[1] Xiaoyang Liu, Minyou Wu. Vehicular CPS: an application of IoT in vehicular networks[J]. Journal of Computer Applications, 2012, 32(4) : $900-904$

[2] Xiao Zhou, Ming Li, Jiajun Bu. The design and implementation of mobile remote medical monitoring system [J]. Computer engineering, 2010, 36 (10): 251253.

[3] Jianqiang Wang, Shiwei Li, Junwei Zeng. Analysis of development model of internet of vehicles [J]. Computer technology and development,2011(12):235-238.

[4] IEEE802. 11p. WAVE-Wireless access for the vehicular environment [S] .2008.

[5] Xiaoping Shi, Airong Huang, Tao Zhang. Research and Development of Sensing Technologies in Internet of Cars [J]. Journal of Hubei Automotive Industries Institute,2011(9):39-44.

[6] Kai Yang, Hanjun Jiang, Zhihua Wang, etc. The Fetal heart rate monitoring system with mobile Internet [J]. Journal of Circuits and Systems (ISCAS), 2014:443-446

[7] Akyildiz I F, Xudong Wang, Weilin Wang. Wireless mesh networks: a survey[J]. Computer Networks, 2005,47(4):445-487. 